\section{Numerical Investigation of Constraint Effects on Ductile Fracture in Tensile Specimens}

This study explores the capabilities of a computational cell framework into a 3-D setting to model ductile fracture behavior in tensile specimens. The cell methodology provides a convenient approach for ductile crack extension suitable for large scale numerical analyses which includes a damage criterion and a microstructural length scale over which damage occurs. Laboratory testing of a high strength structural steel provides the experimental stress-strain data for round bar and circumferentially notched tensile specimens to calibrate the cell model parameters for the material. The present work applies the cell methodology using two damage criterion to describe ductile fracture in tensile specimens: (1) the Gurson-Tvergaard (GT) constitutive model for the softening of material and (2) the stress-modified, critical strain (SMCS) criterion for void coalescence. The present work first applies the cell methodology to investigate effects of constraint (stress triaxiality) on ductile crack initiation of notched tensile specimens. An application also follows to determine the dependence of ductility on stress triaxiality for the tested steel. These exploratory 3-D studies using computational cells clearly demonstrate its capability to predict the strong effects of constraint on measured stress-strain response for tensile specimens.

Keywords: Ductile fracture, tensile specimen, constraint, computational cell, finite elements

\section{Introduction}

Failure assessments of damaged steel components subjected to large scale straining and plastic deformation remain a key issue in design and safety analysis of critical structures, including marine and nuclear facilities, oil and gas pipelines in onshore and offshore systems. Localized yield and subsequent plastic flow caused primarily by extreme or accidental loads potentially cause severe material damage with significant reduction in ductility and fracture toughness. Recent studies conducted after the Northridge earthquake in 1994 (Barson, 2002) and the Kobe earthquake in 1995 (Okashita et al., 1998; Yasuda et al., 2000) have demonstrated the strong effect of large plastic straining induced by ground motion on catastrophic (brittle) failure of welded steel structures. In such structures, fullmoment beam-to-column connections with transverse welds loaded in tension display high constraint and limited ductility. Crack-like defects that developed in these connections appeared after initiation of ductile cracks in highly strained (damaged) regions subjected to normal stresses. During further overload conditions, rapid ductile crack extension of these defects led to complete structural failure. Other typical examples of plastic straining effects on the material degradation process include reeling during the laying operation of steel risers, local buckling of structural components in ships and bridges subjected to overloading, and permanent deformation of buried gas transmission pipelines due to ground slide among others. Consequently, advanced and rational structural analysis procedures must consider the ability of notched components to deform plastically without developing ductile cracks.

Research efforts to model ductile crack initiation in structural components subjected to large plastic deformation have evolved primarily along methodologies using notched tensile bars. These approaches have largely focussed on the effect of the stress state on the effective plastic strain to initiate ductile failure. Early work of Hancock and Mackenzie (1976), Mackenzie et al. (1977) and Beremin (1983) employed notched axisymmetric specimens to measure the ductility levels for a range of structural materials. In particular, the results of Hancock and Mackenzie (1976) led to the

Paper accepted March, 2004. Technical Editor: Edgar Nobuo Mamiya. construction of a failure locus where the strain to initiate cracking by void coalescence in the center of the specimen is a function of the stress triaxiality, $h$, defined by the ratio $h=\sigma_{m} / \sigma_{e}$, where $\sigma_{m}$ is the hydrostatic (mean) stress and $\sigma_{e}$ is the effective Mises stress. These predictions reveal an exponential dependence of ductility on stress triaxiality which compares well with the void growth model of Rice and Tracey (1969).

However, the application of small scale tensile specimens in assessment procedures for ductile crack initiation in larger and more complex structural components requires an understanding of the nonlinear coupling between stress triaxiality and the plastic strain fields. Previous studies (Needleman, 1972, Tvergaard and Needleman, 1984 ) have provided quantitative descriptions of the necking behavior in unnotched tensile specimens; these analyses employ a convenient finite element formulation to describe the onset of necking. In particular, the numerical simulations of necking and failure in a tensile test conducted by Tvergaard and Needleman (1984) incorporate a model for void nucleation into the formulation. Subsequent work by Becker et al. (1988) extends this model to describe void growth in round notched bars. While these results correctly capture the observed ductile failure behavior, studies which employ a micromechanics model based upon a local criterion to predict ductile failure in small scale tensile specimens remain relatively scarce.

This work broadens previous studies on ductile behavior of tensile specimens to address effects of constraint (stress triaxiality) on ductile crack initiation based upon a micromechanics model incorporating void growth (Santos, 2003). The computational cell methodology proposed by Xia and Shih (1995) provides a convenient approach to describe ductile failure within the framework of large scale numerical analyses. These computational cells include a damage criterion and a microstructural length scale over which damage occurs. Ductile crack extension occurs through void growth and coalescence (by cell extinction) within a thin layer of material symmetrically located about the crack plane. An element vanish procedure removes highly voided cells from the analysis thereby creating new traction-free surfaces which extend the macroscopic crack. The cells have initial (smeared) void volume fraction denoted by $f_{0}$. The layer thickness $(D)$ introduces a strong length-scale over which damage occurs; elsewhere, the background 
material obeys the flow theory of plasticity without damage by void growth. The 3-D form of the Gurson-Tvergaard (GT) dilatant plasticity theory (Gurson, 1977; Tvergaard, 1990) provides a suitable description of void growth within the cells. Laboratory testing of a high strength structural steel provides the experimental stress-strain data for round bar and circumferentially notched tensile specimens to calibrate the cell model parameters for the material. The present work first applies the cell methodology to investigate effects of constraint (stress triaxiality) on ductile crack initiation of notched tensile specimens. An application also follows to determine the dependence of ductility on stress triaxiality for the tested steel. These exploratory 3-D studies using computational cells clearly demonstrate its capability to predict the strong effects of constraint on measured stress-strain response for tensile specimens.

\section{Computational Cell Model for Ductile Crack Growth}

\section{Micromechanism of Ductile Fracture}

Ductile fracture in metals is a multistep mode of material failure incorporating the combination of microvoid nucleation, growth and coalescence at the microscale level (see, e.g., the review of Garrison and Moody, 1987). Early experimental studies demonstrated the key role played by microvoid mechanisms on ductile failure of tensile specimens (Tipper, 1949; Puttick, 1959). Microvoids nucleate at inclusions or second-phase particles, either by decohesion of the particle-matrix interface or by fracture of the particle, most often in the center of the neck region as illustrated in Fig. 1(a). Under increased deformation, these microvoids grow until localized plastic flow and necking of the ligament between adjacent microvoids (coalescence of microvoids) create a continuous fracture path (most often assisted by the rapid growth and coalescence of secondary microvoids). Upon further deformation, this internal crack progresses until sufficient loss of cross sectional area leads to final failure of the specimen by a plastic collapse mechanism of the remaining ligament.

Unlike cleavage fracture, which is a mechanism driven almost entirely by the local tensile stresses, inclusion of the microregime of ductile fracture in crack growth analyses is central to relate the material tearing behavior with a macroscopic (engineering) fracture parameter in a continuum framework. However, the complex interplay of the key processes (microvoid nucleation, growth and coalescence) leading to ductile failure underlies the need of additional consideration to describe the micromechanics of ductile tearing. Experimental observations and computational studies show that the plastic strains for nucleation are small thereby causing only little damage in the material ahead of the internal crack formed in the neck region. In contrast, the highly damaged material ahead of the internal crack in the final stages of microvoid coalescence carries only little stresses; the final link-up of microvoids has then a minor effect on the progressive crack growth. Consequently, the kinetics of the (macroscopic) crack extension process of ductile fracture can be considered as driven primarily by the growth of microvoids. Figure 1(b) pictures the schematic path of a growing crack in a ductile material. The material layer enveloping the growing crack, which must be thick enough to include at least a void or microcrack nuclei, identifies a process zone for the ductile fracture which conveniently gives the necessary length dimension for the model. Void growth and coalescence in the layer will cause the surface tractions that the process zone exerts on its surrounding drop to zero (this implicitly defines a traction-separation law for the process zone layer). Micrographs reveal a negligible degree of void growth in material distances from the crack plane of more than 1-2 the spacing of larger inclusions (i.e., in the material outside the planar layer) as indicated in the figure. Although the highly localized path followed by the crack front becomes generally nonplanar and tortuous (alternate sliding-off), macroscopic growth follows a simpler planar character dictated by the symmetric, Mode I loading.
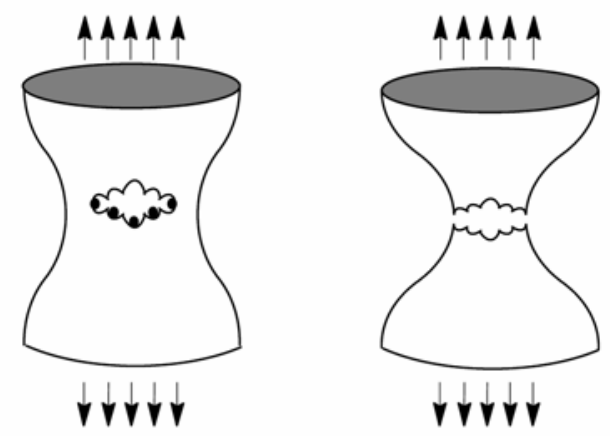

(a)

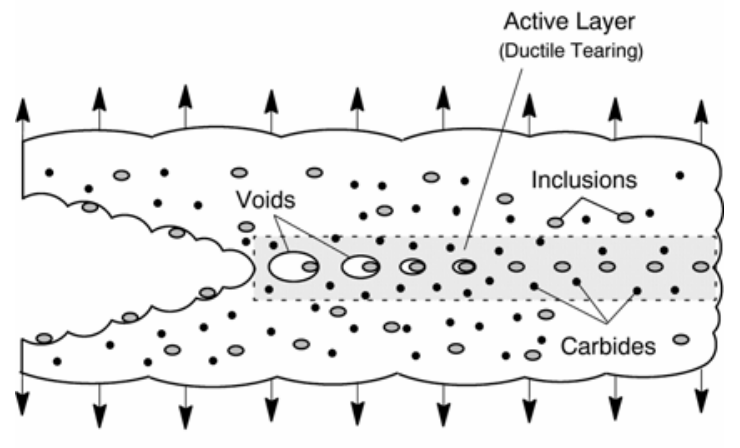

(b)

Figure 1. Phenomenological modeling of ductile fracture. (a) void nucleation and growth in a tensile specimen for a ductile material; (b) schematic path of a growing crack in a ductile material.

\section{Cell Model for Ductile Tearing}

Motivated by the above observations, Xia and Shih (1995) proposed a model using computational cells to include a realistic void growth mechanism, and a microstructural length-scale physically coupled to the size of the fracture process zone. Void growth remains confined to a layer of material symmetrically located about the crack plane, as illustrated in Fig. 2(a), and having thickness $D$, where $D$ is associated with the mean spacing of the larger, void initiating inclusions. This layer consists of cubical cell elements with dimension $D$; each cell contains a cavity of initial volume fraction $f_{0}$ (the initial void volume divided by cell volume). Material outside the computational cells, the "background" material, follows a conventional flow theory of plasticity and remains undamaged by void growth in the cells. As a further simplification, the void nucleates from an inclusion of relative size immediately upon loading. Progressive void growth and subsequent macroscopic material softening in each cell are described with the GursonTvergaard (GT) constitutive model for dilatant plasticity (Gurson, 1977; Tvergaard, 1990) given by

$$
g=\left(\frac{\sigma_{e}}{\sigma_{Y}}\right)^{2}+2 q_{1} f \cosh \left(\frac{3 q_{2} \sigma_{m}}{2 \sigma_{Y}}\right)-\left(1+q_{3} f^{2}\right)=0
$$


where $g$ denotes the yield function, $\sigma_{e}$ denotes the effective Mises (macroscopic) stress, $\sigma_{m}$ is the mean (macroscopic) stress, $\sigma_{Y}$ is the current flow stress of the cell matrix material and $f$ defines the current void fraction. Under multiaxial stress states

$$
\sigma_{e}=\sqrt{3 S_{i j} S_{i j} / 2}
$$

and

$$
\sigma_{m}=\sigma_{k k} / 3=\left(\sigma_{1}+\sigma_{2}+\sigma_{3}\right) / 3
$$

where $S_{i j}$ denotes the deviatoric components of Cauchy stress and $\sigma_{l}$, $l=1,2,3$ are the principal stresses. Factors $q_{1}, q_{2}$ and $q_{3}$ introduced by Tvergaard (1990) in the above Eq. (1) improve the model predictions for periodic arrays of cylindrical and spherical voids; here we use the values $q_{1}=1.25, q_{2}=1.0$ and $q_{3}=q_{1}{ }^{2}$ proposed by Tvergaard. However, Faleskog et al. (1998) also provide additional values of parameters $q_{1}$ and $q_{2}$ for various ranges of material flow properties.

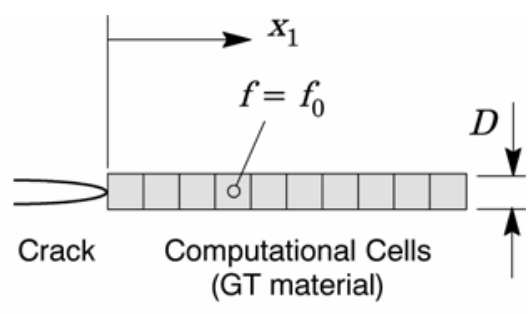

(a)

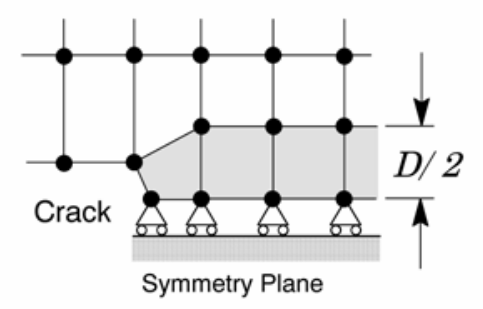

(b)

Figure 2. Modeling of ductile tearing using computational cells.

Figure 2(b) shows the typical, plane strain finite element representation of the computational cell model where symmetry about the crack plane requires elements of size $D / 2$. Material outside the computational cells, the "background" material, follows a conventional flow theory of plasticity and remains undamaged by void growth in the cells. Material properties required for this methodology include: for the background material, Young's modulus $(E)$, Poisson's ratio $(v)$, yield stress $\left(\sigma_{Y}\right)$ and hardening exponent $(n)$ or the actual measured stress-strain curve; and for the computational cells: $D$ and $f_{0}$. The background material and the matrix material of the cells generally have identical flow properties. Using an experimental stress-strain curve obtained from conventional tensile specimens, a series of finite element analyses of the specimen are conducted to calibrate values for the cell parameters $D$ and $f_{0}$ which bring the predicted stress-strain curve into agreement with experiment. Experience with plane-strain finite element analyses of fracture specimens to estimate $D$ and $f_{0}$ for common structural and pressure vessel steels suggests values of 0.05 - $0.2 \mathrm{~mm}$ for $D$ (Ruggieri et al., 1996).

\section{Numerical Crack Growth Using a Cell Extinction Technique}

The GT yield function in Eq. (1) does not model realistically the rapid loss of stress capacity for larger void fractions nearing coalescence levels, nor does the model create new traction free surfaces to represent physical crack extension. In the present work, the evolution of stress within cells follows the original constitutive model of GT in Eq. (1) until $f=f_{E}$, where $f_{E}$ typically has a value in the range $0.15-0.20$. The final stage of void linkup with the macroscopic crack front then occurs by reducing the remaining stresses to zero in a prescribed manner. Tvergaard (1990) refers to this process as the element extinction or vanish technique. The cell extinction process adopted in this work implements a linear-traction separation model (see additional details in Ruggieri et al., 1996). When $f$ in the cell incident on the current crack tip reaches a critical value, $f_{E}$, the computational procedures remove the cell thereby advancing the crack tip in discrete increments of the cell size.

Figure 3 illustrates the cell extinction process coupled with such a linear-traction separation model. This scheme provides computational simplicity while, at the same time, retaining close contact with the physical mechanism of void coalescence just described. Figure 3(a) shows a deformed cell element with initial size normal to the crack (symmetry) plane of $D / 2$; let $H_{0, a v g}$ denote the average elongation of the cell normal to the crack plane as indicated in Fig. 3(a) when the porosity reaches the critical value, $f=$ $f_{E}$. Forces, $\mathbf{P}_{v c}$, exerted on adjacent nodes by the remaining cell stresses are saved and the cell stiffness set to zero (vanished cells remain in the model but are marked inactive). During subsequent load increments, the now vanished cell continues to deform; let $H_{\text {avg }}$ denote the current average (deformed) elongation. The nodal forces $\mathbf{P}_{v c}$ are relaxed to zero in a linear fashion with subsequent increases of $H_{a v g}-H_{0, a v g}$, as shown in Fig. 3(b). At any point after $f=$ $f_{E}$, the remaining fraction of nodal forces applied to the extinct cell is $\gamma \mathbf{P}_{v c}$, with $\gamma$ given by

$$
\gamma=1.0-\frac{H_{a v g}-H_{0, a v g}}{\lambda(D / 2)} \quad, \quad 0 \leq \gamma \leq 1
$$

where a typical value for the release factor, $\lambda$, is 0.1 .

This cell extinction process creates new traction free surfaces in a controlled manner and also eliminates numerical difficulties in the finite strain computations. Cell elements adjacent to the evolving crack front grow increasingly distorted under loading, especially for the small cell sizes commonly used $\left(D \approx 50-200 \times 10^{-3} \mathrm{~mm}\right)$. Compared to plane-strain models, the computations performed by Ruggieri et al. (1996) indicate this problem becomes far more acute in 3-D analyses. Non-uniform growth along the front causes local twisting of elements which would otherwise lead to inadmissible deformation gradients and termination of the analysis. 


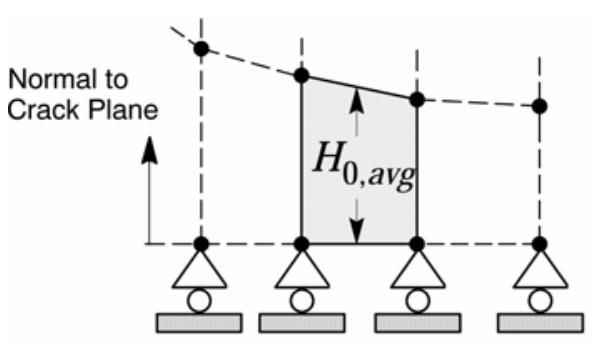

Deformed mesh at $f=f_{E}$

(a)

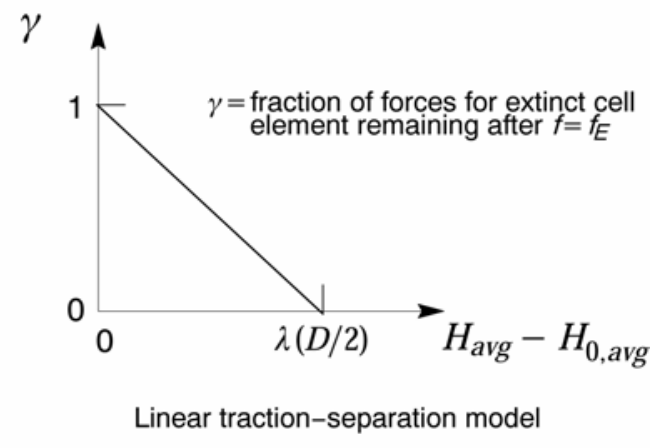

(b)

Figure 3. Schematic illustration of the traction-separation model to release forces of extinct cell elements.

\section{Experimental Measurements}

Toyoda et al. (2000) recently reported on a series of tension tests conducted on a JIS STPT370 pipeline steel. A cylindrical (smooth round bar) and notched tensile specimens with different notch radius were extracted in the longitudinal direction from a $11 \mathrm{~mm}$ thick pipeline (outer diameter $D_{e}=165 \mathrm{~mm}$ ) to measure the stress-strain response of the tested structural steel. The diameter of the central section for the round bar tensile specimen is $6 \mathrm{~mm}$ with a gage length $L=20 \mathrm{~mm}$. The notched specimens have a circumferential notch with a $1 \mathrm{~mm}$ and $2 \mathrm{~mm}$ notch root radius $\left(R_{n}\right)$ and gage length $L=20 \mathrm{~mm}$; the diameter of the central section for this specimen (deepest point of the notch root) is also $6 \mathrm{~mm}$. These notched tensile specimens are denoted R1 and R2 specimens. Figure 4 presents the geometry and dimensions of the tensile specimens used in the experiments. The material has $315 \mathrm{MPa}$ yield stress $\left(\sigma_{Y}\right)$ at room temperature $\left(20^{\circ} \mathrm{C}\right)$ and relatively high hardening properties defined by the ration between the tensile strength, $\sigma_{t}$, and the yield stress for the round bar specimen given by $\sigma_{t} / \sigma_{Y} \approx 1$.4. Figure 5 shows the measured true stress-logarithmic strain curve for the round bar and the notched tensile specimens.
To investigate the mechanism of ductile crack initiation and propagation, a number of tension tests were interrupted at several strain levels and the specimens were unloaded. These unloaded specimens were sectioned near the center plane (longitudinal symmetry) followed by preparation and polishing of the sectioned surface. Laboratory observation using an optical microscope revealed the onset of ductile tearing and growth of the (macroscopic) crack with increased levels of applied strains at the center of the specimen sections. Moreover, post-test fractographs of the fracture surface for the specimens loaded until final failure clearly showed equiaxed dimples in the central region of the fractured specimens with a large number of small microvoids nucleated in the material surrounding the larger microvoids (nucleated from the larger inclusions dispersed in the matrix). Toyoda et al. (2000) present additional details of the experimental program which provide strong support to use the micromechanics approach for ductile failure based upon the cell model adopted in this study.

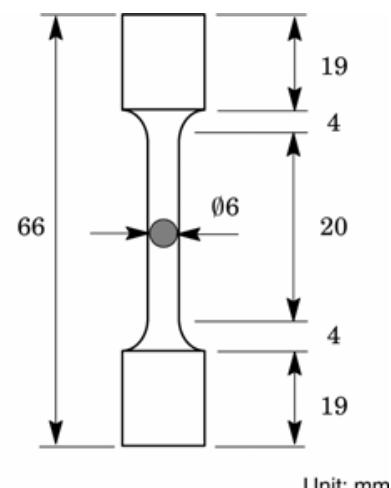

(a)

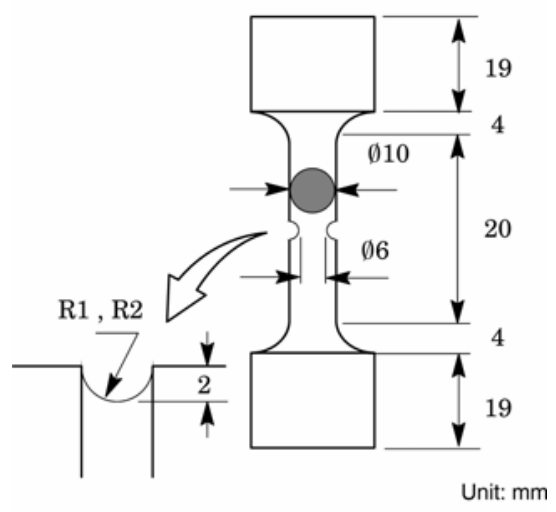

(b)

Figure 4. Geometry of tensile specimen employed in the experiments: (a) round bar specimen; (b) circumferentially notched specimen. 


\section{$\sigma(\mathrm{MPa})$}

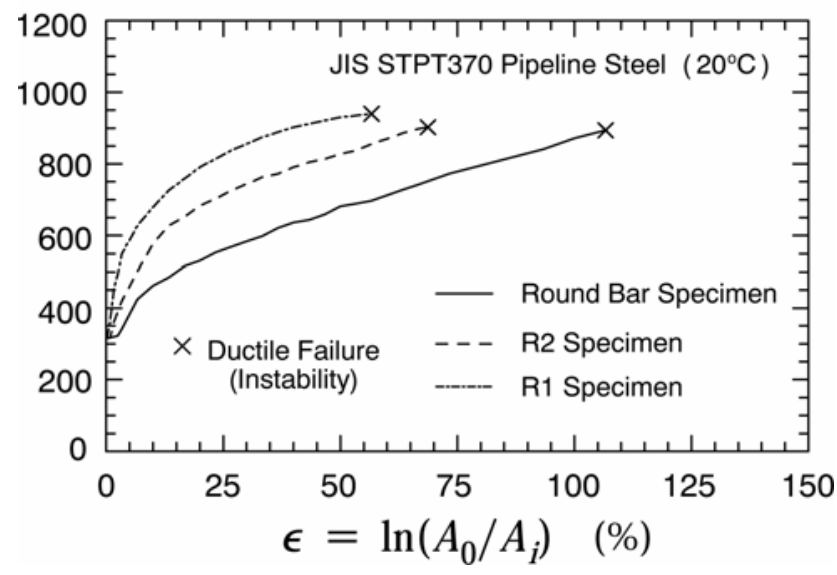

Figure 5. Experimentally measured true stress - logarithmic strain curve for the round bar and circumferentially notched tensile specimens (Toyoda et al, 2000).

\section{Computational Procedures}

\section{Finite Element Models}

Figures 6(a-b) show the finite element models constructed for 3D analyses of the cylindrical tensile specimens (round bar and R2 notched specimen) utilized to measure the stress-strain response for the structural steel employed in this study. The geometry and size of the models match those for specimens tested in the experiments previously described (see Fig. 4). To decrease execution time, the 3D finite element models do not include modeling of the specimen rigid grip shown in Fig. 4; numerical analyses indicate a negligible effect of this region on the tensile response for the specimens. The rigid grip conditions are modelled by imposing $X$-direction and $Y$ direction constraints on the external surface of the outermost layer $(Z=L / 2)$. Symmetry of the geometry and loading conditions enables analyses using only one-quarter, 3-D model of the specimens. Appropriate constraints are imposed on the symmetry planes. Displacement controlled loading $(\Delta)$ of the models as indicated in Fig. 6 permits continuation of the analyses once the load decreases during necking.

The 3-D model for the round bar tensile specimen has 13860, 8node elements arranged into 22 variable thickness layers over the half-length $(L / 2)$, as illustrated in Fig. 6(a). The first layer arranged on the central region of the specimen $(Z=0)$ contains a square of $400(20 \times 20)$ computational cells (each one modelled by a cubic element of dimension $D / 2 \times D / 2 \times D / 2$ ). This layer enables modeling of the planar ductile crack extension well at the longitudinal center plane (neck region). The 3-D model for the circumferentially notched specimen (R2) has 24470, 8-node elements arranged into 28 variable thickness layers over the half-length $(L / 2)$, as illustrated in Fig. 6(b). The first layer contains an approximate square with 474 computational cells to model planar ductile crack extension well at the longitudinal center plane (deepest point of the notch region). Each cell is also modelled by a cube of dimension $D / 2 \times D / 2 \times D / 2$ in a similar arrangement for the round bar tensile specimen to accommodate the notch root geometry (see Fig. 4).

The analyses utilize a piecewise-linear approximation of the measured true stress-logarithmic strain curve for the round bar tensile specimen shown in Fig. 4, but with the uniaxial true stress corrected for necking effects on plastic flow using Davidenkov's equation (see additional details in Toyoda et al, 2000). Other mechanical properties needed for the analyses include $E=206 \mathrm{GPa}$ and $v=0.3$. The matrix material of the computational cell elements and the void-free background material are assigned these properties.

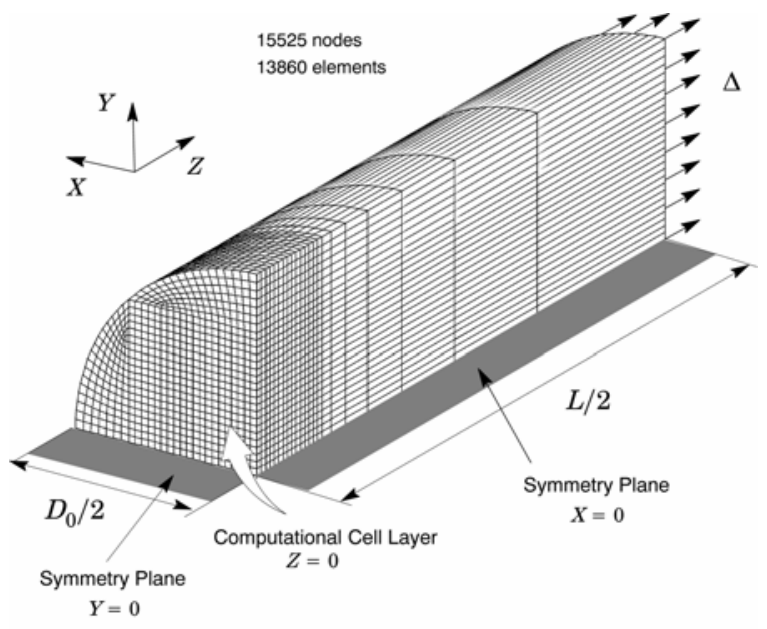

(a)

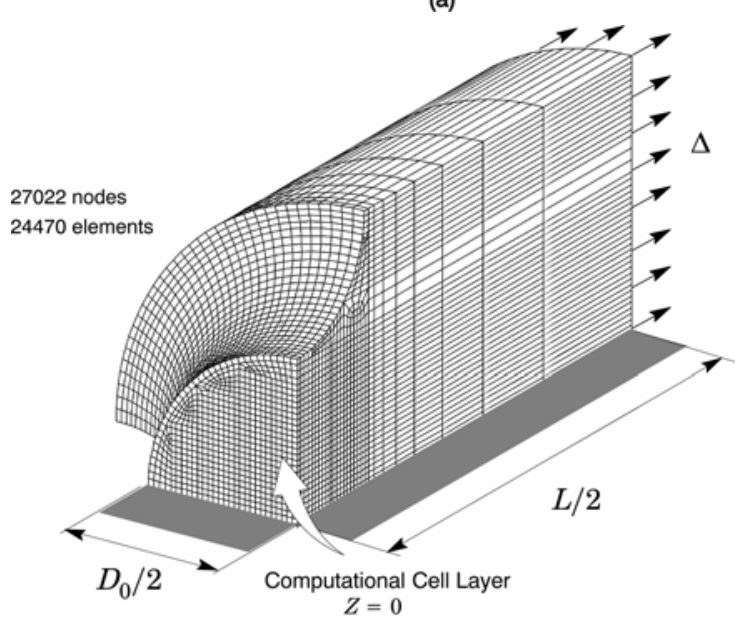

(b)

Figure 6. Finite element 3-D models for the tensile specimens employed in the analyses: (a) round bar specimen; (b) circumferentially notched specimen).

\section{Numerical Solutions}

Finite element solutions are generated using the WARP3D code (Koppenhoefer et al., 1994) which: (1) implements the GT and Mises constitutive models in a 3-D finite-strain framework, and (2) provides automatic cell extinction coupled to the GT model. The nonlinear implementation of the finite element method in WARP3D employs a continuously updated formulation naturally suited for solid elements having only translational displacements at the nodes. The governing equations of equilibrium derive from the principle of virtual work expressed on the current configuration at step $k+1$

$$
\int_{A_{K+1}} \mathbf{T}_{k+1} \cdot \delta \mathbf{u}_{k+1} d A-\int_{V_{K+1}} \delta \mathbf{e}: \mathbf{S}_{k+1} d V=0
$$

where $\mathbf{S}_{\kappa+1}$ denotes the Cauchy stress, $\mathbf{T}$ defines the applied surface tractions, $\delta \mathbf{u}$ defines an admissible virtual displacement field acting on the model at $k+1$, and $\delta \mathbf{e}$ represents the symmetric rate of the 
virtual deformation tensor relative to the current configuration (see Malvern, 1969). $V_{k+1}$ and $A_{k+1}$ denote the current deformed volume and surface area, respectively. Starting from Eq. (5) linearized about the current configuration, the global solution proceeds in an incremental-iterative (implicit) manner with nodal equilibrium stringently enforced at $k+1$. Full Newton iterations advance the solution from $k \rightarrow k+1$. An extrapolation scheme to estimate the displacement increment for the step and the use of consistent tangent moduli for the GT and Mises constitutive models prove essential to maintain rapid convergence of the iterations. Final increments of logarithmic strain over $k \rightarrow k+1$ are then computed using the linear strain-displacement matrix evaluated on the converged mid-increment configuration, $\mathbf{x}_{k+1 / 2}$.

To accommodate finite strains and rotations, the GT and Mises constitutive equations are formulated using strains-stresses and their respective rates defined on an unrotated frame of reference, computed from polar decompositions of the deformation gradients. The stress-update proceeds using a conventional small-strain, backward Euler procedure as described by Healy and Dodds (1992). The polar decompositions insure accuracy in the rotational operations independent of the displacement gradient magnitudes over $k \rightarrow k+1$. The implementation of the backward Euler integration scheme for the GT model builds upon Aravas's work (Aravas, 1987). The linearized form of Eq. (5) requires a tangent operator which couples the spatial rates of Cauchy stress and deformation tensor. The procedure adopted here follows that described by Nagtegaal and Veldpaus (1984), which uses the exact consistent tangent operator on the unrotated configuration and the Green-Naghdi rate of the spatial Cauchy stress.

WARP3D analyzes fracture models constructed with threedimensional, 8-node hexahedral elements. Use of the so-called $\mathbf{B}_{b a r}$ formulation (Hughes, 1980) precludes mesh lock-ups that arise as the deformation progresses into fully plastic, incompressible modes. Dilatational terms of the original strain-displacement matrix, $\mathbf{B}^{\text {dil }}$, are replaced by a volume averaged set of dilatational terms, $\left(\mathbf{B}^{\text {dil }}\right)_{\text {avg }}$, which yield uniform mean stress over the element and minimal locking. The $\mathbf{B}$ matrix thus has the form $\mathbf{B}_{b a r}=\mathbf{B}^{\text {dev }}+\left(\mathbf{B}^{\text {dil }}\right)_{a v g}$, where $\mathbf{B}^{d e v}$ denotes the unmodified deviatoric contribution. Stabilization to prevent hourglass modes takes the form $\mathbf{B}_{b a r}=\mathbf{B}^{d e v}+\left(\mathbf{B}^{d i l}\right)_{a v g}+\xi[$ $\left.\mathbf{B}^{\text {dil }}-\left(\mathbf{B}^{\text {dil }}\right)_{\text {avg }}\right]$ with $\xi$ typically 0.10 .

\section{Calibration of Cell Parameters}

The cell size $D$ and initial porosity $f_{0}$ define the key parameters coupling the physical and computational models for ductile crack growth at the neck region of the tensile specimens. To apply the cell model to a specific material, these two parameters must be calibrated to bring the numerical predictions into agreement with experimental measurements. These parameters should not be viewed as metallurgical parameters representing the microscopic observations of void spacing and initial void volume fraction but rather as computational parameters phenomenologically calibrated. Here, we choose the cell parameters $D$ and $f_{0}$ which provide the best fit to the experimentally measured stress-strain data for the material.

The approximate measure of spacing between the large inclusions for common pressure vessel steels provides values for $D$ in the 0.05-0.2 mm range (Xia and Shih, 1995; Ruggieri et al., 1996). Such range of values should also be applicable to the modeling of ductile failure behavior in tensile specimens using the computational cells. While the mapping of one finite element per cell must provide adequate resolution of the stress-strain fields in the active layer and in the adjacent background material, the numerical stress-strain response of the tensile specimen scales almost proportionally with $D$ for a fixed $f_{0}$ (a thicker layer requires more total work to reach critical conditions). Consequently, to avoid potential numerical difficulties with too low $f_{0}$-values while, at the same, using a sufficiently refined mesh, the present work adopts $D=0.2 \mathrm{~mm}$ as the cell size parameter employed in the numerical analyses. With parameter $D$ determined, the initial volume fraction, $f_{0}$, remains as the only parameter unspecified. The calibration process then focuses on determining a suitable value for the initial volume fraction, $f_{0}$, that produces the best fit to the measured tensile data for the material.

Figure 7 shows the measured and predicted true stresslogarithmic strain curves for the round-bar tensile specimen with $\varepsilon$ defined by $\ln \left(A_{0} / A_{i}\right)$, where $A_{0}$ is the initial cross sectional area and $A_{i}$ is the current (instantaneous) cross sectional area (neck region). Predicted curves are shown for four values of the initial volume fraction, $f_{0}=0.0075,0.01,0.0135$ and 0.015 . For all $f_{0}$-values, the predicted tensile response agrees well with the measured values up to strain levels of $\approx 100 \%$. However, the prediction of instability by ductile failure (point marked $x$ ) in the specimen varies widely with initial volume fractions; this point follows the onset of rapid crack growth in the internal region of neck section with a marked decrease of the load carrying capacity of the specimen. For $f_{0}=0.0135$, the predicted instability point agrees well with the experimental measure. In contrast, the use of $f_{0}=0.0075,0.01$ and 0.015 predicts the instability for the specimen at very different strain levels.

\section{$\sigma(\mathrm{MPa})$}

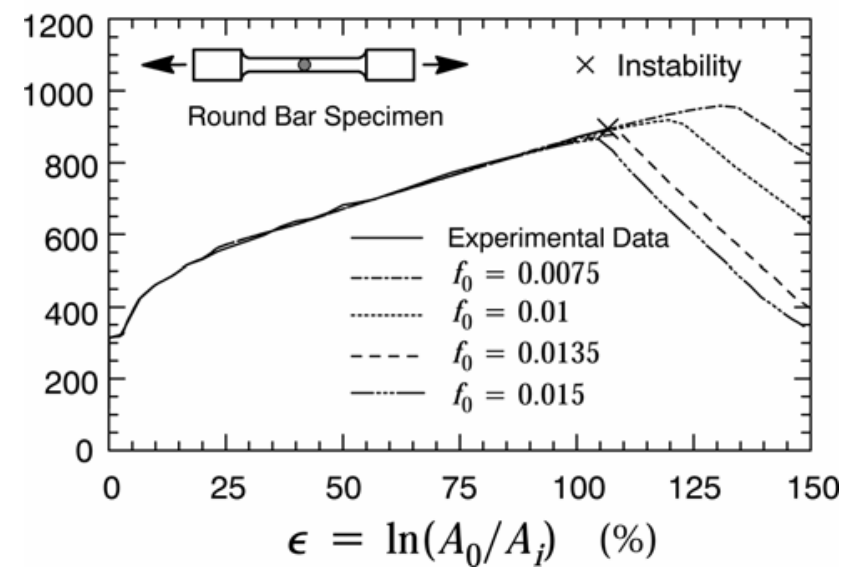

Figure 7. Comparison of measured and predicted true stress - logarithmic strain curve for the round bar tensile specimen.

Figures 8 and 9 show the measured and predicted true stresslogarithmic strain curves for the circumferentially notched (R2 and R1) tensile specimen with $\varepsilon$ defined by $\ln \left(A_{0} / A_{i}\right)$. The numerical analyses for these specimens also adopt $D=0.2 \mathrm{~mm}$ as the cell size parameter. The numerical results are shown for $f_{0}=0.01,0.004$ and 0.005 . In both plots, the predicted tensile responses agree well with the measured values up to strain levels of $\approx 40 \%$ for all $f_{0}$-values but we note that the numerical predictions provide slightly higher stress values for a fixed strain level for the R2 specimen. Such behavior is most likely associated with discrepancies between the (numerical) elastic-plastic hardening model and the actual hardening behavior for the material at higher strains. Most importantly, however, the prediction of instability by ductile failure (point marked $x$ ) in the specimens also varies widely with initial volume fractions; this point follows the onset of rapid crack growth with a marked decrease of the load carrying capacity for these specimens. Here, the predicted instability point agrees well with the experimental measures of both specimens for $f_{0}=0.004$. 
$\sigma(\mathrm{MPa})$

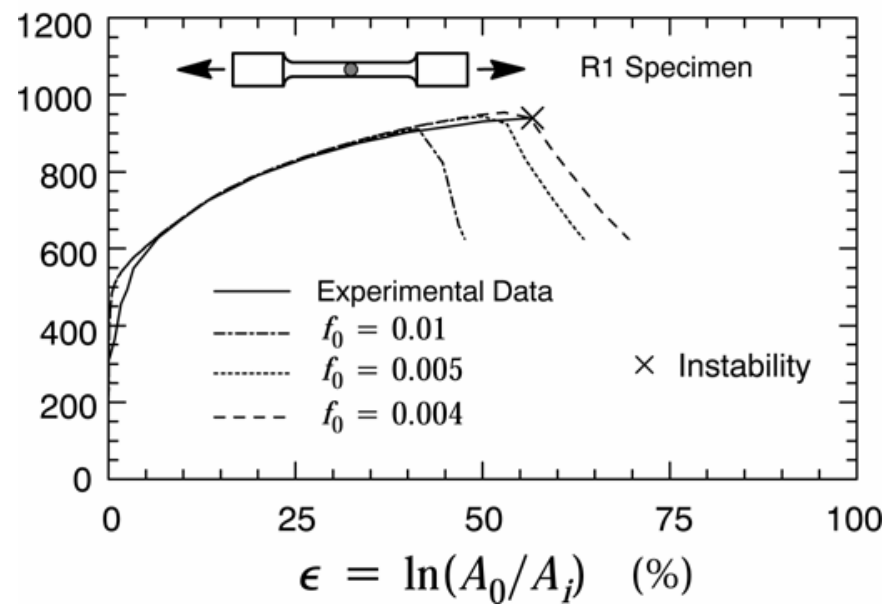

Figure 8. Comparison of measured and predicted true stress - logarithmic strain curve for the R1 notched specimen.

$\sigma(\mathrm{MPa})$

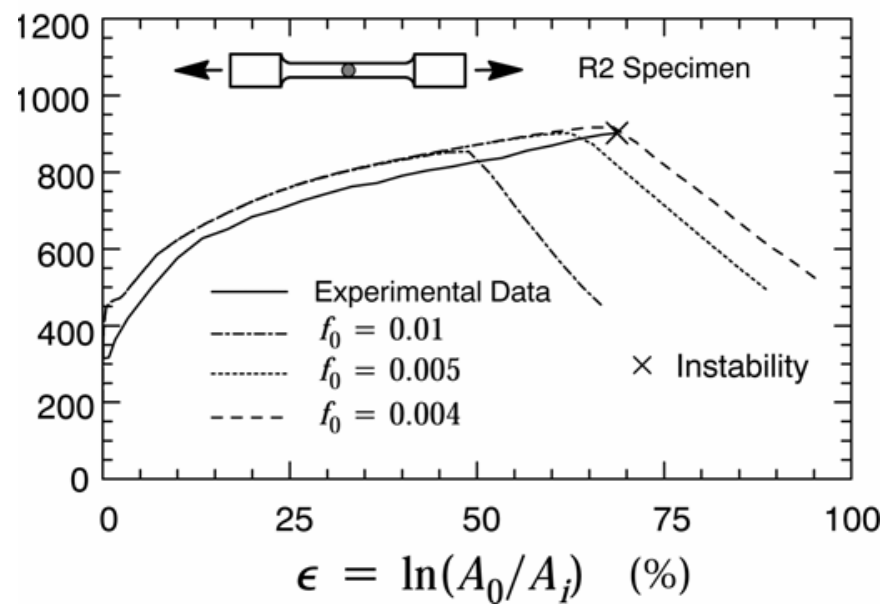

Figure 9. Comparison of measured and predicted true stress - logarithmic strain curve for the R2 notched specimen.

\section{Effects of Constraint on Stress and Strain Behavior}

Constraint most generally refers to the evolving level of stress triaxiality ahead of a notch front under increased remote loading. A widely adopted approach to describe the levels of constraint (stress triaxiality) which develop in notched specimens upon increased loading defines a constraint parameter, $h$, as the ratio of mean (hydrostatic) stress, $\sigma_{m}$, to Mises stress, $\sigma_{e}$, i.e., $h=\sigma_{m} / \sigma_{e}$. Since parameter $h$ varies with position ahead of the notch, it reflects the mechanism of ductile fracture by void growth in the present context (recall that the rate of void growth depends exponentially on the hydrostatic stress as indicated by the yield function, Eq. (1), previously described).

Figure 10 shows the distribution of stress triaxiality, $h$, at the specimen centerplane $(Z=0)$ with radial distance (measured from specimen center), $R$, for the round bar tensile specimen. Plots are shown at three loading levels: $\varepsilon=5 \%$, maximum load (which corresponds to a deformation level $\approx 20 \%$ for this specimen and marks the beginning of necking) and load corresponding to the onset of crack growth at the internal region of neck section. The results indicate: (1) at $\varepsilon=5 \%$ and maximum load, the stress triaxiality is nearly invariant along the specimen cross section, and (2) stress triaxiality at the onset of crack growth increases significantly in the internal region of the specimen cross (neck) section.

$$
h=\sigma_{m} / \sigma_{e}
$$

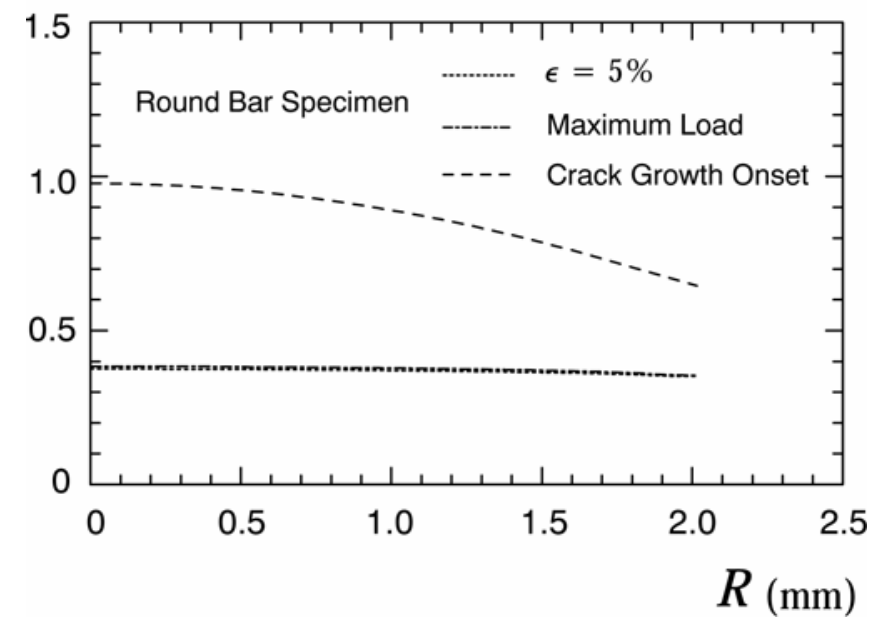

Figure 10. Distribution of stress triaxiality, $h$, with radial distance, $R$, for the round bar tensile specimen.

Additional insight into the effects of increased stress triaxiality can be gained by examining the necking process in the central region of the specimen. Figures 11(a-b) present key results obtained from numerical simulation of the ductile behavior for the round bar tensile specimen using the cell model with the calibrated cell parameter, $f_{0}=0.0135$. With increased deformation after the initiation of necking in the specimen $(\approx 20 \%)$, the axial load begins to fall below the peak load due to increased damage in material of the internal region at the central section. After intense necking develops in the specimen, final failure occurs at strain level $\varepsilon \approx 105 \%$ as illustrated by the deformed profile of Fig. 11(a). During this process, higher values of stress triaxiality develop at the center region of the specimen cross section causing extensive local crack growth (notice the strong effect of $\sigma_{m}$ in the GT potential function, Eq. (1), on reducing the material stress capacity during void growth). Figure 11(b) shows a snapshot of the (macroscopic) internal crack at the instability point $(\varepsilon \approx 105 \%)$. For consistency, the location of the growing crack tip in the plane-strain analyses is taken at the cell with $f=0.1$. This corresponds to a position between the cell currently undergoing extinction and the peak stress location; at this position stresses are decreasing rapidly and the void fraction is increasing sharply; Xia and Shih (1995) discuss this issue in detail. Here, the internal crack has extended $\approx 1.4 \mathrm{~mm}(\approx 14$ cells in the $X$-direction and $Y$-direction) before final failure. When an instability point is eventually reached (point marked as $\times$ in Fig. 7), the remaining material in the specimen ligament cannot keep pace with the increased deformation so that specimen collapse occurs. Unfortunately, the comparison of the predicted size for the internal crack at ductile failure with experimental measurements cannot be made here - such measurements are not available. Nevertheless, this trend of void growth development is entirely consistent with the experimental observations reported by Toyoda et al (2000) in that the internal crack is formed in the center of the neck region and progresses towards the specimen surface. 


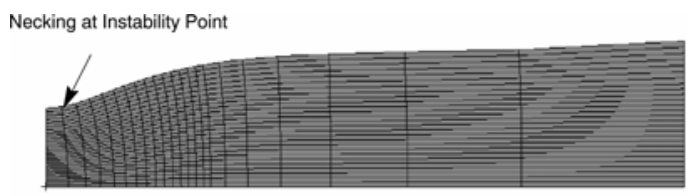

(a)

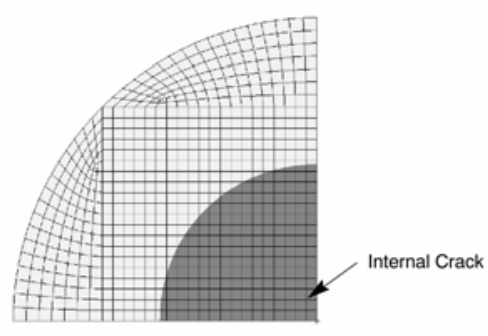

(b)

Figure 11. Evolution of necking and void growth of the round bar tensile specimen for the analysis using $\mathrm{f} 0=0.0135$ : (a) necking at final failure for $\varepsilon=105 \%$; (b) internal crack formed by void growth at $\varepsilon=105 \%$.

Figures 12 and 13 provide the distribution of stress triaxiality, $h=\sigma_{m} / \sigma_{e}$, at the specimen centerplane $(Z=0)$ with radial distance (measured from specimen center), $R$, for the R2 and R1 specimens. Plots are also shown at three loading (deformation) levels: $\varepsilon=5 \%$, maximum load and load corresponding to the onset of crack growth at the internal region of neck section. As could be expected, the results show a significant elevation of stress triaxiality for the notched specimens at all load levels as compared to the stress triaxiality levels for the round bar specimen. Moreover, for every load level considered, the increase in stress triaxiality is much more pronounced for the R1 specimen (note that the scales of the plots are different). These levels of stress triaxiality, $h$, correlate directly with the numerical predictions previously displayed in Figs. 8 and 9; notice again the key role played by $h$ in the evolution of void growth expressed by the GT potential function, Eq. (1). The numerical results also predict the growth of an internal crack in the center of the notch (neck) region for both specimens, which progresses towards the specimen surface (to conserve space, they are not shown here). This behavior again follows the same trends previously described for the round bar specimen and entirely agrees with the experimental observations reported by Toyoda et al. (2000).

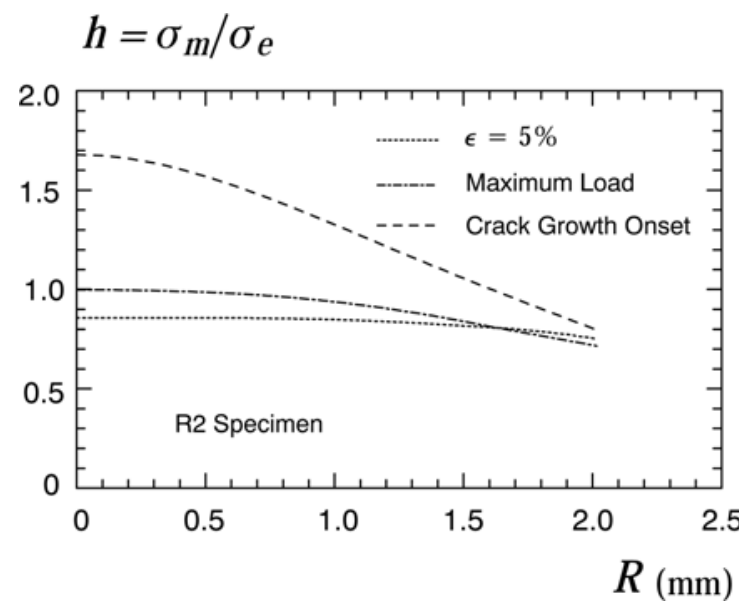

Figure 12. Distribution of stress triaxiality, $h$, with radial distance, $R$, for the $\mathbf{R} 2$ tensile specimen.

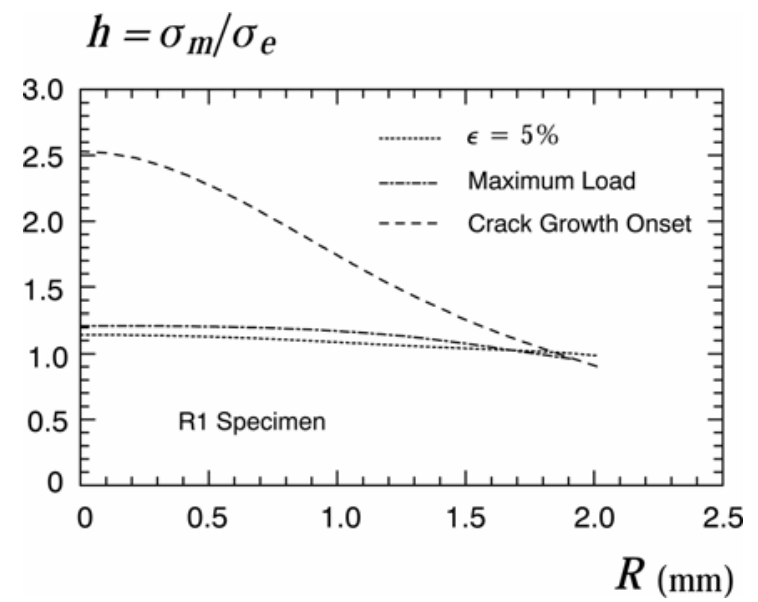

Figure 13. Distribution of stress triaxiality, $h$, with radial distance, $R$, for the R1 tensile specimen.

\section{Ductile Failure Locus}

The previous results demonstrated the strong effect of constraint (stress triaxiality) on ductile fracture for the tensile specimens with varying notch geometry. Earlier experimental studies on the correlation of failure strains with the stress state ahead of notches in high strength structural steels led Hancock and Mackenzie (1976) to propose a simplified criterion to describe ductile failure in terms of a stress triaxiality parameter and the effective plastic strain at failure initiation. Based upon the Rice and Tracey (1969) model for void growth, Hancock and Mackenzie (1976) provide the critical plastic fracture strain, $\varepsilon_{p, c r i t}$, in the form

$$
\varepsilon_{p, c r i t}=\alpha \exp \left(-\beta \frac{\sigma_{m}}{\sigma_{e}}\right)
$$

where $\alpha$ and $\beta$ are material constants calibrated from experimentally measured tensile data. Equation (6) defines a failure locus for the material which represents all critical combinations of plastic strain, $\varepsilon_{p}$, and stress triaxiality $\left(\sigma_{m} / \sigma_{e}\right)$ leading to ductile failure.

Panontin and Shepard (1995) describe a complete study of the calibration process to estimate $\alpha$ and $\beta$ from notched-tensile data for an A516 pressure vessel steel and an HY 80 steel. In their work, Panontin and Shepard refer to Eq. (6) as the stress-modified, critical strain (SMCS) criterion for void coalescence. However, such study employs a conventional elastic-plastic analysis to determine the plastic strain at failure which does not consider material damage nor crack growth. The present work defines the critical combination of equivalent plastic strain $\left(\varepsilon_{p}\right)$ and stress triaxiality $\left(h=\sigma_{m} / \sigma_{e}\right)$ for the damaged material at the onset of crack growth at the internal region of neck section. Operationally, $\varepsilon_{p}$ and $h$ are taken at the cell with current porosity $f=0.1$ for both conditions which corresponds to a position between the cell currently undergoing extinction and the peak stress location as previously discussed. Fig. 14 presents the variation of stress triaxiality with equivalent plastic strain for the tested material using the crack growth analyses for the tensile specimens. The calibrated parameters which define the ductile failure locus for the tested steel are $\alpha=1.77$ and $\beta=0.51$.

\section{Concluding Remarks}

This study describes a 3-D computational framework to model ductile failure behavior in cylindrical tensile specimens. Material separation and formation of an internal (macroscopic) crack occurs 
through a local fracture mechanism based on the growth and coalescence of microvoids dispersed in the material. The GursonTvergaard dilatant plasticity model for voided materials describes the eventual loss of material stress capacity under sustained loading. Fixed-size computational cell elements defined on a thin layer adjacent to the crack plane provide an explicit length scale for the continuum damage model. An element vanish procedure removes highly voided cells from further consideration in the analysis, thereby creating new traction-free surfaces which extend the macroscopic crack. The key micromechanics parameters are $D$, the thickness of the computational cell layer, and $f_{0}$, the initial cell porosity. These parameters are calibrated through numerical analyses to match the stress-strain response obtained by testing conventional tensile specimens. Such calibration scheme provides computational parameters which are loosely coupled, at best, with metallurgical features of the material.

The 3-D analyses of round bar and circumferentially notched tensile specimens demonstrate the capability to predict the tensile response and the ductile behavior including the evolution of void growth. In particular, numerical results for the tensile specimens analyzed in this work predict the instability point (ductile failure) in very good agreement with experimental measurements. The computations predict remarkably well the development of necking in the specimens and the growth of a macroscopic crack at the internal region of neck section prior to instability. The present work also demonstrates the strong effect of specimen constraint on ductile failure. The 3-D analyses reveal increased stress triaxiality at specimen center with increased deformation which has important implications on the phenomenology of ductile failure for notched structurals components. While additional experimental and numerical studies are needed to further validate applications of the cell model to predict ductile failure in larger structures, the present study, when taken together with previous analyses (Xia and Shih, 1995; Ruggieri et al., 1996; Gao et al, 1998), provide an additional body of results against which the robustness of the cell model approach can be weighed. Ongoing work is currently underway to extend the model to correlate ductile cracking in damaged pipelines subjected to large scale straining.

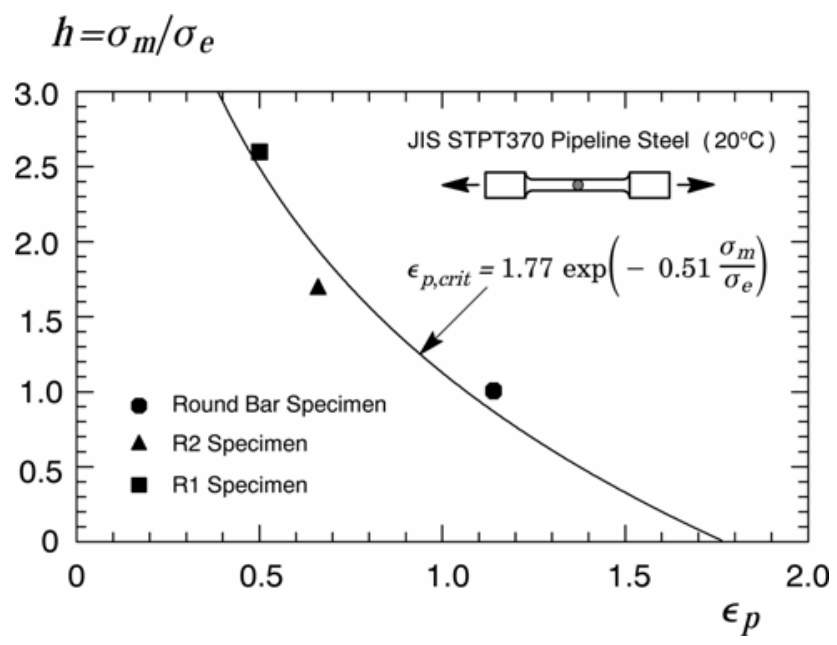

Figure 14. Ductile failure locus for the tested steel.

\section{Acknowledgements}

This investigation was supported by Fundação de Amparo à Pesquisa do Estado de São Paulo (FAPESP) through Grant 01/06919-9. Funding to the author is also provided by Conselho Nacional de Desenvolvimento Científico e Tecnológico (CNPq).
The many valuable discussions and contributions from Prof. Masao Toyoda and Dr. Mitsuru Ohata (Osaka University) are also gratefully aknowledged.

\section{References}

Aravas, N., 1987, “On the Numerical Integration of a Class of PressureDependent Plasticity Models,” International Journal for Numerical Methods in Engineering, Vol. 24, pp. 1395-1416. .

Barson, J. M., 2002, "Fatigue and Fracture Behavior of Moment Frame Connections Under Seismic Loading (Northridge Earthquake,” in Fatigue and Fracture Mechanics: 33rd Volume, ASTM STP 1417, R. S. Piascik and W. G. Reuter, Eds., American Society for Testing and Materials, Philadelphia, pp. 57-72.

Becker, R., Needleman, A., Richmond, O. and Tvergaard, V, 1988, "Void Growth and Failure in Notched Bars", Journal of the Mechanics and Physics and Solids, Vol. 36, pp. 317.

Beremin, F.M., 1983, “A Local Criterion for Cleavage Fracture of a Nuclear Pressure Vessel Steel,” Metallurgical Transactions, Vol. 14A, pp. 2277-2287.

Faleskog, J., Gao, X. and Shih, C. F., 1998, “Cell Model for Nonlinear Fracture Analysis - Micromechanics Calibration,” International Journal of Fracture, Vol. 89, pp. 375-398.

Gao, X. Falescog, J., Dodds, R. H., and Shih, C. F., 1998, "Ductile Tearing in Part-Trough Surface Cracks: Experiments and Cell Model Predictions” Engineering Fracture Mechanics, Vol. 59, pp. 761-770.

Garrison, W. M, Jr. and Moody, N. R., 1987, “Ductile Fracture,” Journal of the Physics and Chemistry of Solids, Vol. 48, pp. 1035-1074.

Gurson, A. L., 1977, “Continuum Theory of Ductile Rupture by Void Nucleation and Growth: Part I - Yield Criteria and Flow Rules for Porous Ductile Media,” Journal of Engineering Materials and Technology, Vol. 99, pp. 2-15.

Hancock, J.W. and Mackenzie, A. C., 1976, "On the Mechanism of Ductile Failure in High-Strength Steels Subjected to Multiaxial StressStates”, Journal of Mechanics and Physics of Solids, Vol. 24, pp. 147-169.

Healy, B.E. and Dodds, R..H., 1992, “A Large Strain Plasticity Model for Implicit Finite Element Analyses,” Computational Mechanics, Vol. 9, No. 2, pp. 95-112

Hughes, T. J., 1980, "Generalization of Selective Integration Procedures to Anisotropic and Nonlinear Media,” International Journal for Numerical Methods in Engineering, Vol. 15, pp. 1413-1418. .

Koppenhoefer, K., Gullerud, A., Ruggieri, C., Dodds, R. and Healy, B., 1994, "WARP3D: Dynamic Nonlinear Analysis of Solids Using a Preconditioned Conjugate Gradient Software Architecture”, Structural Research Series (SRS) 596, UILU-ENG-94-2017, University of Illinois at Urbana-Champaign. .

Mackenzie, A. C., Hancock, J.W. and Brown, D. K. 1977, "On the Influence of State of Stress on Ductile Failure Initiation in High Strength Steels”, Engineering Fracture Mechanics, Vol. 9, pp. 167-188.

Malvern, L., 1969, "An Introduction to the Mechanics of Continuum Media”, Prentice-Hall, New Jersey.

Nagtegaal, J. C. and Veldpaus, F. E., 1984, "On the Implementation of Finite Strain Plasticity Equations in a Numerical Model,” In Numerical Analysis of Forming Processes (edited by J.F. Pittman, O. C. Ziekiewicz, R. D. Wood and J. M. Alexander), p. 351. John Wiley and Sons, New York.

Needleman, A., 1972, "A Numerical Study of Necking in Circular Cylindrical Bars”, Journal of the Mechanics and Physics and Solids, Vol. 20, pp. 111-127.

Okashita, K., Ohminami, R., Michiba, K., Yamamoto, A., Tomimatsu, M., Tanji, Y. And Miki, C., 1998, "Investigation of the Brittle Fracture at the Corner of P75 Rigid-Frame Pier in Kobe Harbor Highway During the Hyogoken-Nanbu Earthquake”, Journal of the Japan Society of Civil Engineers, Vol. 591-I43, pp. 243.

Panontin, T. L. and Sheppard, S. D., 1995, “The Relationship Between Constraint and Ductile Fracture Initiation as Defined by Micromechanical Analyses” in Fracture Mechanics: 26th Volume, ASTM STP 1256 (W. G. Reuter, J. H. Underwood and J. C. Newman, Jr., Eds.), pp. 54-85.

Puttick, K. E., 1959, "Ductile Fracture in Metals," Philosophical Magazine, Vol. 4, pp. 964-969.

Rice, J. R. and Tracey, D. M., 1969, “On the Ductile Enlargement of Voids in Triaxial Stress Fields”, Journal of the Mechanics and Physics and Solids, Vol. 17, pp. 201-217.

Ruggieri, C., Panontim, T. and Dodds, R. H., 1996, "Numerical Modeling of Ductile Crack Growth in 3-D Using Computational Cell Elements,"’'International Journal of Fracture, Vol. 82, pp. 67-95, 1996. 
Santos, F. F., 2003, “A Micromechanics Approach to Ductile Fracture in Structural Steels and Applications to Failure Analysis in Pipelines”, MSc Thesis, Polytechnic School, Faculty of Engineering, University of Sao Paulo. Tvergaard, V., 1990, "Material Failure by Void Growth to Coalescence,” Advances in Applied Mechanics, Vol. 27, pp. 83-151, 1990.

Tvergaard, V. and Needleman, A., 1984, "Analysis of Cup-Cone Fracture in a Round Tensile Bar”, Acta Metallurgica, Vol. 32, pp. 157.

Tipper, C. F., 1949, “The Fracture of Metals,” Metallurgia, Vol. 39, pp. 133-137, 1949.

Toyoda, M., Ohata, M, Ayukawa, N., Ohwaki, G., Ueda, Y and Takeuchi, I., 2000, "Ductile Fracture Initiation behavior pf Pipe Under a
Large Scale Cyclic Bending” in Pipeline Technology, Vol. II (R. Denys, Ed.), pp. 87-102.

Yasuda, O., Hirono, M., Ohata, M. and Toyoda, M., 2000, "Ductile Crack Initiation Behavior of Pre-Strained Steel,” International Institute of Welding, IIW Doc. X-1461

Xia, L. and Shih, C. F., 1995, "Ductile Crack Growth - I. A Numerical Study Using Computational Cells with Microstructurally-Based Length Scales," Journal of the Mechanics and Physics of Solids, Vol. 43, pp. 233259. 